\title{
Desempeño del pavimento con mezcla reciclada-RAP y grano de caucho reciclado-GCR
}

\section{Pavement performance with reclaimed asphalt pavement-RAP and recycled tire rubber-RTR}

Ana Sofía Figueroa Infante. I.C., Ph.D. Investigación en Desarrollo Tecnológico, INDETEC Universidad de la Salle, Bogotá-Colombia

afigueroa@unisalle.edu.co

\author{
Elsa Fonseca Santanilla. Química. Doctora en Agrociencias. \\ Investigación en Desarrollo Tecnológico, INDETEC \\ Universidad de la Salle, Bogotá-Colombia \\ elsafonseca@unisalle.edu.co
}

Fecha de recepción: 25 de marzo de 2020 / Fecha de aprobación: 13 de julio de 2020

\section{RESUMEN}

Esta investigación muestra los resultados del desempeño del pavimento flexible (ahuellamiento y fatiga) usando pavimento asfáltico recicladoRAP y grano de caucho reciclado-GCR. Los dos tipos de materiales son residuos que provienen de diferentes procesos: el primero proviene del reciclaje de pavimento asfáltico y el segundo de las llantas en desuso por desgaste de diferentes vehículos. Los estudios incluyeron el análisis de los materiales en la condición original y una propuesta de su mejoramiento, considerando la disminución de la producción de gases efecto invernadero. El producto obtenido en este proyecto pretende ser utilizado en la red vial secundaria y terciaria de Colombia, para mejorar la conexión entre zonas de producción agrícola y los centros urbanos de tal manera que se contribuye con el mejoramiento y desarrollo de la competitividad de estas zonas.

Palabras clave: RAP, GCR, Fatiga, Ahuellamiento, RCD en carreteras.

\begin{abstract}
This research shows the results on the flexible pavement performance (rutting and fatigue) using Reclaimed Asphalt Pavement-RAP and Recycled Tire Rubber. The two types of materials are waste that comes from different processes: the first one comes from recycling asphalt pavement and the second from disused tires due to wear and tear of different vehicles. The studies include analysis of the materials in the original condition and a proposal for improving the material, considering the reduced production of greenhouse gases. The product obtained in this project intended to be used in secondary and tertiary roads (rural roads) in Colombia, to improve the connection between agricultural production areas and urban centers in such a way that it contributes to the improvement and development of the competitiveness of these areas.
\end{abstract}

Keywords: RAP, RTR, Fatigue, Rutting, CEDW in roads. 


\section{INTRODUCCIÓN}

El reciclaje de pavimento es una técnica ampliamente conocida que requiere de un rejuvenecedor para su reutilización (Minnesota Department of Transportation, 2002) y las investigaciones recientes se han centrado especialmente en la optimización de los porcentajes de Reclaimed Asphalt Pavement (RAP) permitidos para la fabricación de mezcla nueva (Fernández del Campo, 2003). Los estudios sobre la utilización del RAP datan de los años 80 con apoyo del Departamento de Transporte de Illinois y la Universidad de Illinois, quienes desarrollaron una metodología de diseño e investigaron el aporte del asfalto residual en estas mezclas (Illinois Center for Transportation, 2007). Durante los años sucesivos las diferentes investigaciones han centrado sus esfuerzos en la posibilidad de utilización del máximo porcentaje posible de RAP en mezclas nuevas sin afectar su desempeño. Estos porcentajes típicamente se han conservado en el 30\% de RAP con el 70\% de material virgen. Sin embargo, hay países como Holanda en el que se tienen experiencias con porcentajes superiores.

En Colombia se han realizado estudios puntuales sobre el uso del RAP, para responder al compromiso del país con la sostenibilidad ambiental. Es así como en la Universidad de la Salle en el año 2009 (Espinosa, 2009) se realizó una investigación con el apoyo de la alcaldía de Engativá para optimizar, en términos del proceso constructivo, la utilización del RAP proveniente de las vías locales de la zona en mención, la cual tenía los acopios de RAP copados y sin posibilidad de uso. En esta investigación quedaron abiertas las opciones para seguir investigando sobre el uso RAP en el país. Otras investigaciones al respecto se han venido realizando por algunas universidades e instituciones gubernamentales (Ostos et al., 2012).

Como parte esencial del estudio del RAP, el análisis de la adhesión, la cohesión, la fatiga y el ahuellamiento del material son necesarias para estudiar su desempeño bajo cargas vehiculares, ya que estos materiales han pasado por un proceso que ha alterado sus propiedades originales y, por lo tanto, requieren estudios adicionales para su utilización, (Figueroa, Velásquez, Reyes, y Bahía, 2013; Herrera, Velásquez, Infante, Gómez, y Lizcano, 2013; Qin, Schabron, Boysen, y Farrar, 2014).

En el año 2011, la Federal Highway Administration (FHWA) publicó un documento oficial sobre el estado de la práctica del reciclaje en los Estados Unidosylas metodologías para realizarlo (Federal Highway Administration, 2011). En tal documento se resalta la efectividad del costo, la responsabilidad ambiental y el buen desempeño, como aspectos esenciales que un buen proceso de reciclaje debe satisfacer. Adicionalmente, se indica que es necesario promover la utilización de la mayor cantidad de RAP, pero garantizando el mismo o mejor desempeño que tiene una mezcla asfáltica completamente virgen.

En el Cuadro 1 se presenta la cantidad de RAP utilizado para diferentes proyectos en los Estados Unidos.

\section{Cuadro 1. Proyectos de campo utilizando altos RAP}

\begin{tabular}{|c|c|c|}
\hline Lugar & $\%$ RAP & Fecha de construcción \\
\hline North Carolina & $40 \%$ & Sep-07 \\
\hline South Carolina & 30 y $50 \%$ & Oct-07 \\
\hline Wisconsin & $25 \%$ & Nov-07 \\
\hline Florida & $45 \%$ & Dec-07 \\
\hline Kansas & 30 a $40 \%$ & May-08 \\
\hline Delaware & $35 \%$ & verano 2008 \\
\hline Minnesota (MnROAD) & $30 \%$ & 2008 \\
\hline Illinois & $\begin{array}{c}10 \text { a } 50 \% \\
\text { permitido }\end{array}$ & 2008 \\
\hline
\end{tabular}

Fuente: Federal Highway Administration (2019)

\section{MARCO TEÓRICO}

La determinación de la fatiga de las mezclas asfálticas permite encontrar la deformación máxima de la misma antes de que ocurra la falla a un número de ciclos de carga determinado. Este análisis predice la vida útil de la mezcla asfáltica. Este daño ocurre en las capas ligadas de pavimento flexible y se presenta cuando se generan valores elevados de deformación a tracción en la zona inferior de la capa asfáltica, producto de las cargas repetitivas (Hsu y Tseng 1996; Masad, Muhunthan, Shashidhar, y Harman, 1998).

Según la literatura, la definición para el fenómeno de fatiga de mezclas asfálticas es un proceso de cambio estructural permanente y progresivo, que ocurre en un punto del material sujeto a esfuerzos y deformaciones de amplitudes variables y tiene como resultado la aparición de fisuras que conducen a una fractura total del material, debido a la aplicación de un número determinado de repeticiones o ciclos (Rondón, Reyes, Gónzalez, y Vásquez, 2012). De acuerdo con Lundstrom, Di Benedetto, y Isacsson (2004), se reconocen dos fases de daño: en la primera, conocida como la fase de iniciación, hay aparición de microfisuras generando disminución en la rigidez de la estructura; en la segunda fase, llamada de propagación, hay unión de microfisuras ocasionando que éstas sean cada 
vez mayores y que se propaguen rápidamente. Según el mismo autor, los principales factores que inciden en la respuesta a la fatiga de las mezclas asfálticas son: el método de compactación de la muestra, el modo de carga y el tipo de ensayo aplicado, los parámetros de diseño de la mezcla y las variables asociadas al ambiente.

De acuerdo con Tangella, Craus, Deacon, y Monismith (Strategic Highway Research Program, 1990), los métodos de ensayo que se emplean para medir el comportamiento de la fatiga pueden agruparse en las siguientes categorías: ensayos de flexión simple, ensayos de flexión con apoyos, ensayos axiales directos, ensayos diametrales, ensayos triaxiales y ensayos de pistas. Los ensayos de laboratorio han verificado que la relación entre la deformación $\varepsilon$ (producida por la carga) y la duración o vida de fatiga del material, representada por el número, $\mathrm{N}$, de veces que soporta la carga antes de romperse por fatiga están ligadas por la Ecuación 1.

$$
N=K_{1} * \varepsilon^{K_{2}}
$$

donde

$N$ : Número de ciclos de carga hasta la fatiga del material.

$\varepsilon$ : Deformación unitaria de tracción (en micro deformaciones, $\mu m / m$ ).

$K_{1}$ y $K_{2}$ : Constantes que describen el comportamiento a fatiga del material.

Estos últimos se ha comprobado que están principalmente afectados por el módulo de la mezcla, el contenido de asfalto, la viscosidad del asfalto, la granulometría y la naturaleza de los agregados, el contenido de aire y la temperatura del pavimento.

El conocer el comportamiento a fatiga de las mezclas bituminosas es básico para proceder al dimensionamiento del pavimento en los métodos analíticos. La falla por fatiga fue observada en primer lugar en los materiales metálicos, cuando se sometían a una tensión inferior a la de rotura y se fisuraban si se aplicaba la solicitación repetidamente. Desde los primeros estudios sobre este tipo de fisura por fatiga en metales se dedujo que podía establecerse una relación logarítmica entre la tensión o deformación impuesta y el número de solicitaciones, como se muestra a continuación (Ecuaciones 2 y 3 ).

$$
\begin{aligned}
& \log N \varepsilon=a-(b * \log \varepsilon) \\
& \log N \sigma=c-(d * \log \sigma)
\end{aligned}
$$

donde

$N \varepsilon, N \sigma$ : Número de aplicaciones de carga aplicados en un ensayo de fatiga a deformación o tensión controlada.

$\varepsilon$ : deformación impuesta, en un ensayo de fatiga a deformación controlada.

$\sigma$ : tensión impuesta, en un ensayo de fatiga a tensión controlada.

$a, b, c y d$ : Características del material ensayado.

\section{MATERIALES Y MÉTODOS}

La selección de las muestras de RAP se consiguió a través del Instituto de Desarrollo Urbano-IDU, entidad gubernamental administradora de la red vial en Bogotá con dominio y potestad del material RAP, quienes tienen acopios de diferentes proyectos de la ciudad en donde se están llevando a cabo procesos de mejoramiento vial a partir del fresado y reemplazo de la carpeta asfáltica. Los materiales se clasificaron en cinco grupos con similitud visual homogénea (Figura 1). La selección de los acopios para esta fase de la investigación se realizó con base en la especificación I.N.V. E-201-07.

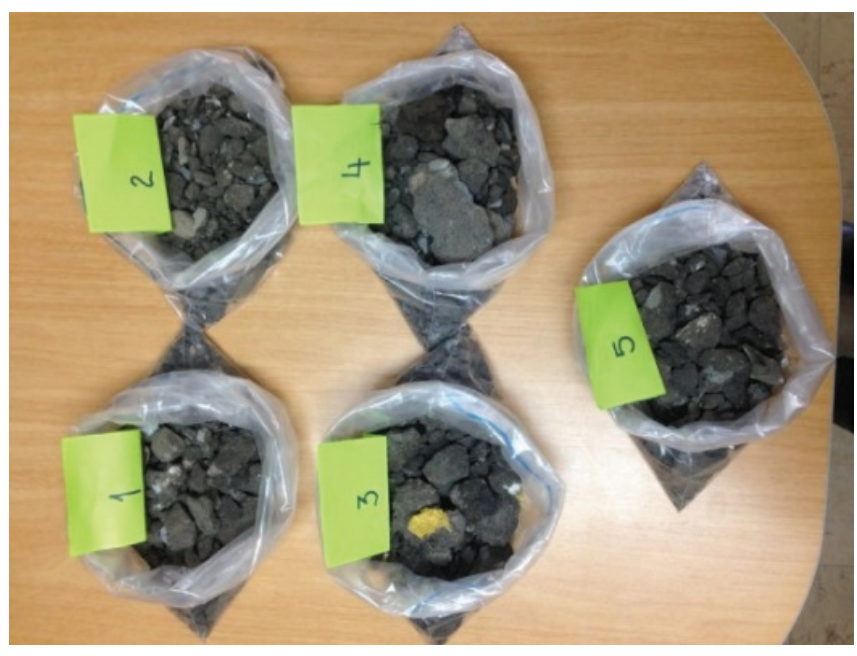

Figura 1. Muestras de RAP de los diferentes acopios 
Se realizó la granulometría del material RAP original (Cuadro 2) para determinar la distribución de los agregados y su correspondencia con las mezclas de rodadura especificadas en la norma colombiana. La distribución gráfica de estos agregados se observa en la Figura 2.

Como se observa en la Figura 2, la granulometría no cumple con las especificaciones del límite inferior, de tal manera que se realizó un arreglo granulométrico al RAP con agregado virgen para dar cumplimiento a la granulometría más cercana al RAP utilizado para la investigación. De acuerdo con los tamaños obtenidos durante el fresado, se escogió la Mezcla Densa en Frío con tamaño máximo nominal 25mm (MDF-25) según I.N.V. E-440-13. Ver Cuadro 3.

Cuadro 2. Granulometría del RAP original

\begin{tabular}{|c|c|c|c|c|c|c|}
\hline Tamiz & Abertura $(\mathrm{mm})$ & Peso $(\mathrm{g})$ & $\%$ Retenido & \% Pasa & $\begin{array}{c}\text { Límite } \\
\text { Inferior }\end{array}$ & Limite Superior \\
\hline 1 & 25,00 & 0,00 & 0,00 & 100,00 & 100 & 100 \\
\hline $3 / 4$ & 19,00 & 600,90 & 6,49 & 93,51 & 80 & 95 \\
\hline $1 / 2$ & 12,50 & 1273,60 & 13,75 & 79,77 & 0 & 0 \\
\hline $3 / 8$ & 9,50 & 678,80 & 7,33 & 72,44 & 69 & 75 \\
\hline No. 4 & 4,75 & 1782,50 & 19,24 & 53,20 & 47 & 62 \\
\hline No. 8 & 2,26 & 1661,90 & 17,94 & 35,26 & 35 & 50 \\
\hline No. 50 & 0,30 & 2348,20 & 25,35 & 9,91 & 13 & 23 \\
\hline No. 200 & 0,08 & 853,70 & 9,22 & 0,70 & 3 & 8 \\
\hline Fondo & & 64,40 & 0,70 & 0,00 & & \\
\hline
\end{tabular}

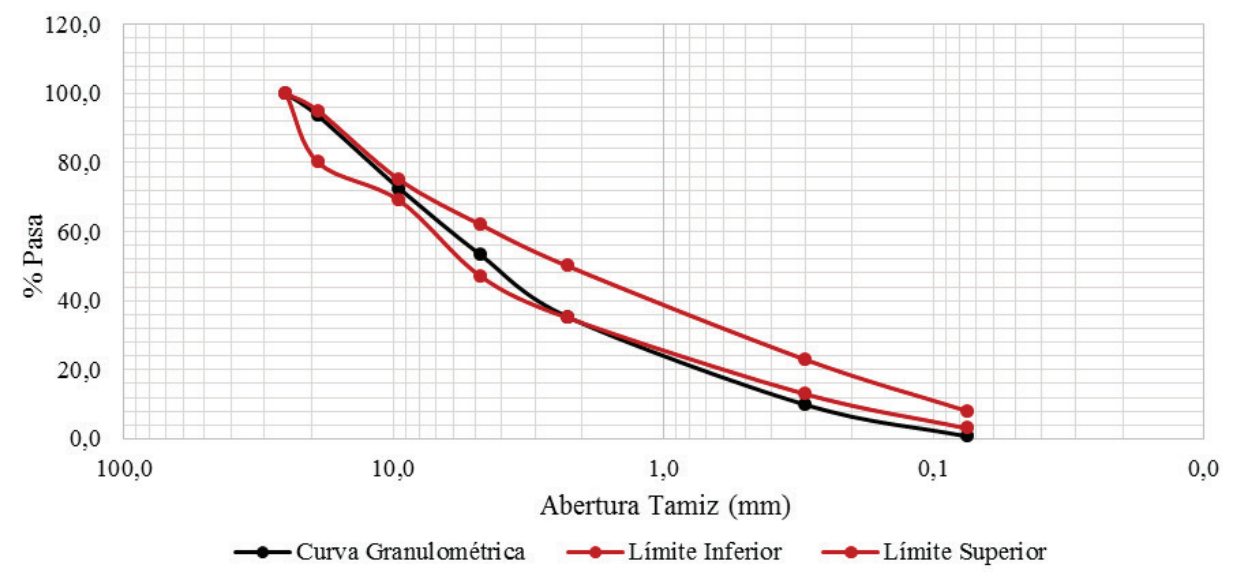

Figura 2. Curva granulométrica del material RAP original 


\begin{tabular}{|c|c|c|c|c|c|c|}
\hline Tamiz & Abertura (mm) & Peso (gr) & $\%$ Retenido & $\%$ Pasa & Límite Inferior & Límite Superior \\
\hline 1 & 25 & 0,00 & 0,00 & 100,00 & 100 & 100 \\
\hline $3 / 4$ & 19 & 87,50 & 12,50 & 87,50 & 80 & 95 \\
\hline $1 / 2$ & 12,5 & 54,21 & 7,74 & 79,75 & 0 & 0 \\
\hline $3 / 8$ & 9,5 & 51,31 & 7,33 & 72,42 & 69 & 75 \\
\hline No. 4 & 4,75 & 134,76 & 19,25 & 53,17 & 47 & 62 \\
\hline No. 8 & 2,26 & 74,69 & 10,67 & 42,50 & 35 & 50 \\
\hline No. 50 & 0,3 & 171,50 & 24,50 & 18,00 & 13 & 23 \\
\hline No. 200 & 0,075 & 88,20 & 12,60 & 5,40 & 3 & 8 \\
\hline \multicolumn{2}{|c|}{ Fondo } & 37,80 & 5,40 & 0,00 & & \\
\hline \multicolumn{2}{|c|}{ Total } & 699,97 & & & & \\
\hline
\end{tabular}

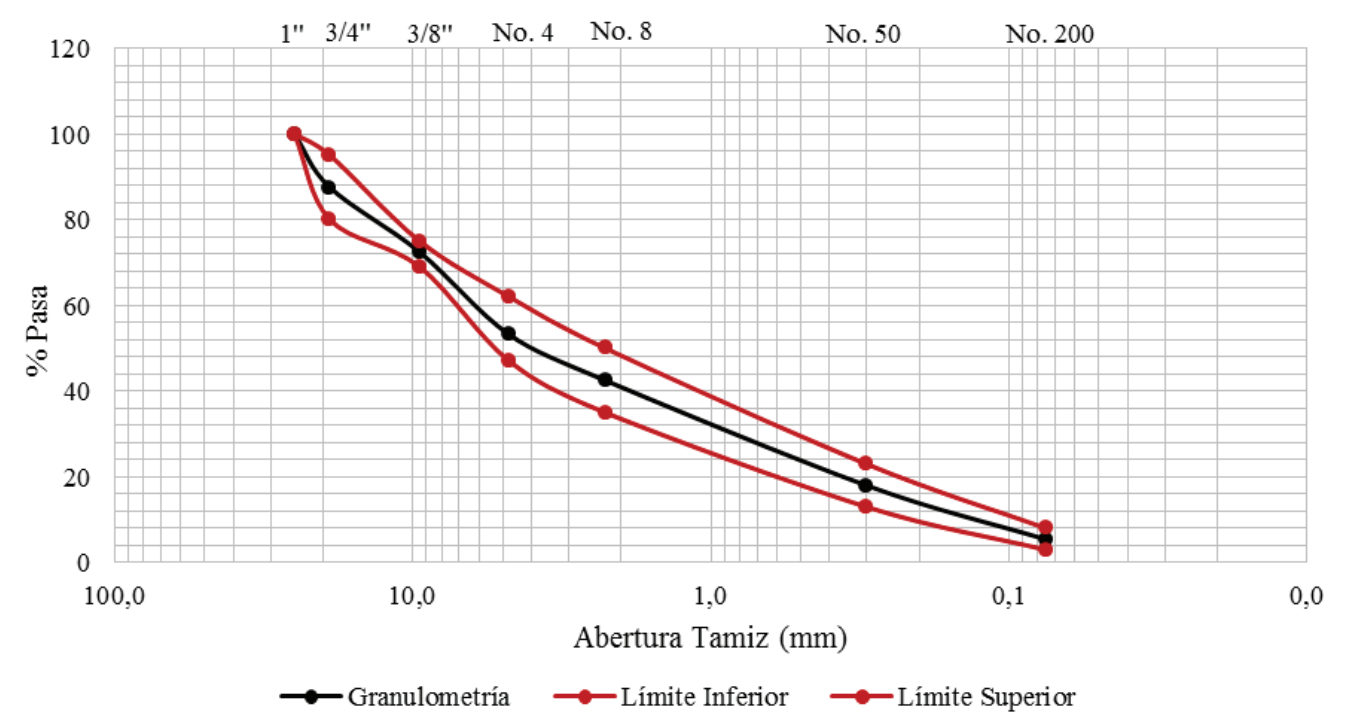

Figura 3. Curva granulométrica del RAP mejorada con agregado virgen

\section{DISCUSIÓN DE RESULTADOS}

\subsection{Fatiga}

En la presente investigación se realizó el ensayo de fatiga con muestras trapezoidales, en cuyo caso el comportamiento a la fatiga se estudia por medio de ensayos de flexión realizados a $25^{\circ} \mathrm{C}$ y $10 \mathrm{~Hz}$.

El principio del ensayo se basa en someter una probeta trapezoidal a un desplazamiento sinusoidal de amplitud constante en la parte superior. Las probetas deben estar sujetas en su base inferior; en la base superior se les aplica un tren de on- das continuas sinusoidales a una amplitud dada. La ruptura se define en el momento en que la fuerza que se aplica en el extremo de la probeta se reduce a la mitad de la inicial para un desplazamiento dado.

Es necesario evaluar varias amplitudes del desplazamiento para determinar la pendiente de la ley de fatiga (b), y el valor de la deformación admisible, $\varepsilon_{6}$, para 1.000.000 de Hertz.

Las probetas son de forma trapezoidal con altura de $250 \mathrm{~mm}$, espesor de $25 \mathrm{~mm}$ y bases mayor y menor de $70 \mathrm{~mm}$ y $25 \mathrm{~mm}$, respectivamente, y compactada en el equipo universal garantizando la densidad obtenida en el diseño volumétrico de la mezcla. Ver Figura 4. 


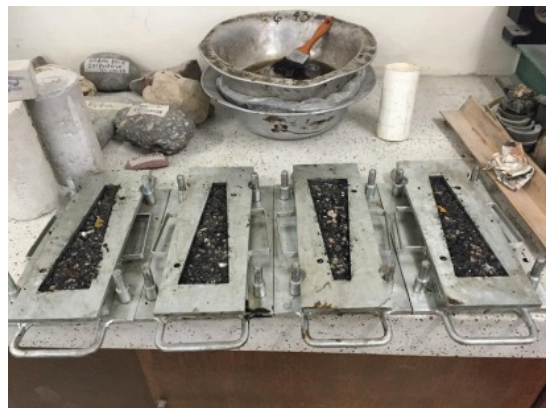

(a)

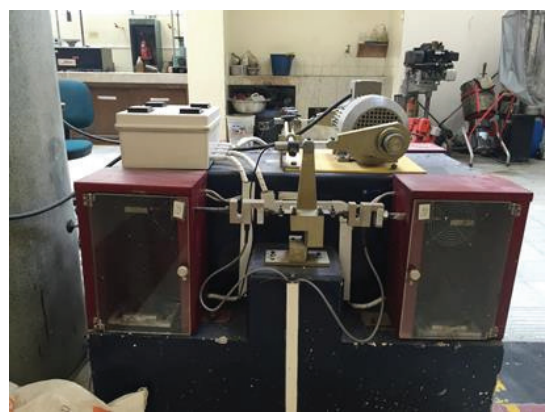

(b)

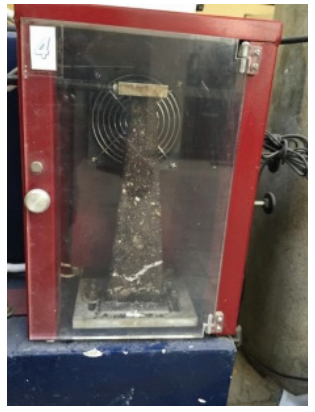

(c)

Figura 4. (a) Elaboración de las briquetas trapezoidales, (b) equipo de ensayo, (c) falla de la briqueta RAP original

La ley de fatiga del material RAP original para una Mezcla Densa en Frío (MDF-25) se realizó con base en la norma francesa NFP 98 261-1 a una temperatura ambiente de laboratorio $\left(20^{\circ} \mathrm{C}\right)$, teniendo en cuenta tres deformaciones controladas de 90, 150 y $220 \mu \mathrm{m}$. Ver la Figura 5.
Para analizar la diferencia en fatiga del material RAP en contraste con una mezcla modificada con emulsión asfáltica Catiónica de Rompimiento Lento (CRL-1) y Grano de Caucho Reciclado (GCR), se realizó el mismo proceso. Ver la Figura 6.

Ley de fatiga material RAP 100\%

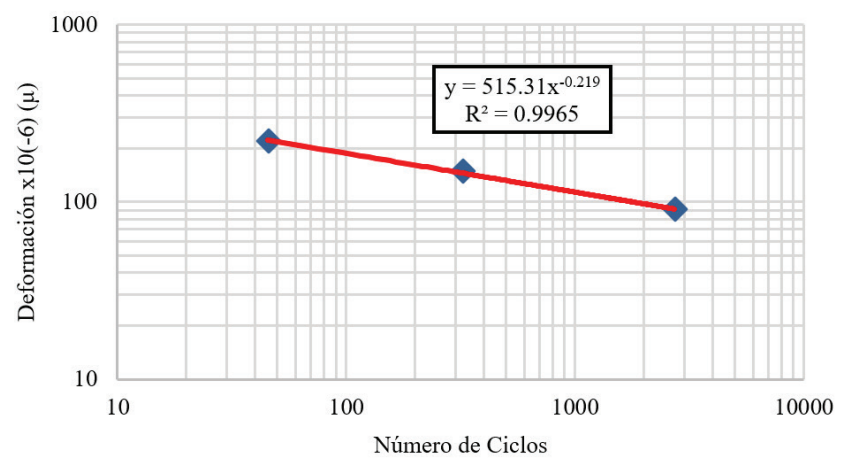

Figura 5. Ley de fatiga del material RAP original

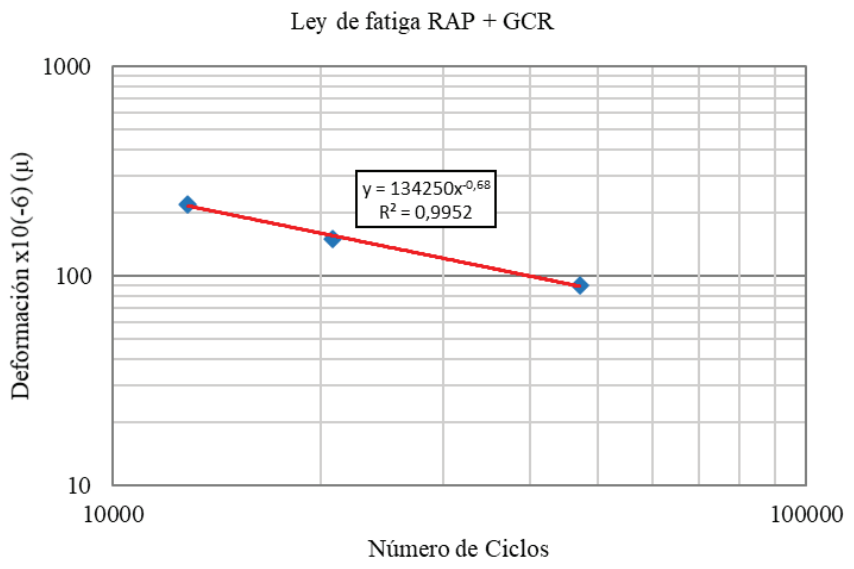

Figura 6. Ley de fatiga para la mezcla RAP+GCR 
La comparación de la ley de fatiga de las dos mezclas, 100\% RAP y RAP+GCR, evidencia la diferencia de pendientes entre las dos, de tal manera que, para un millón de ciclos, la mezcla $100 \%$ RAP presenta mayor deformación respecto a la modificada con emulsión CRL-1 y GCR.

\subsection{Ahuellamiento}

Para la determinación del ahuellamiento se construyó el equipo descrito en la norma INV E 756 13, y se realizaron las briquetas para tres ensayos y dos briquetas por cada uno, según esta especificación. Al momento de presentar este artículo se tiene la curva de ahuellamiento para el material modificado con emulsión asfáltica y caucho reciclado. Sin embargo, se están realizando muestras de contraste para tener información suficiente de repetitividad de los ensayos de deformación plástica. Ver la Figura 7.

Para la determinación del desempeño bajo las solicitaciones esperadas se diseñó y construyó el equipo de ahuellamiento, de acuerdo con la especificación INV E 756-13. Con el propósito de someter el material a condiciones extremas para determinar su respuesta, se consideró alto tráfico. Ver la Figura 8.

La deformación plástica para este primer set (seis briquetas) según la INV E 756-13, arrojó resultados de 0,34 cm de deformación máxima, sin embargo, este dato debe ser contrastado con más muestras y diferentes contenidos de asfalto residual.
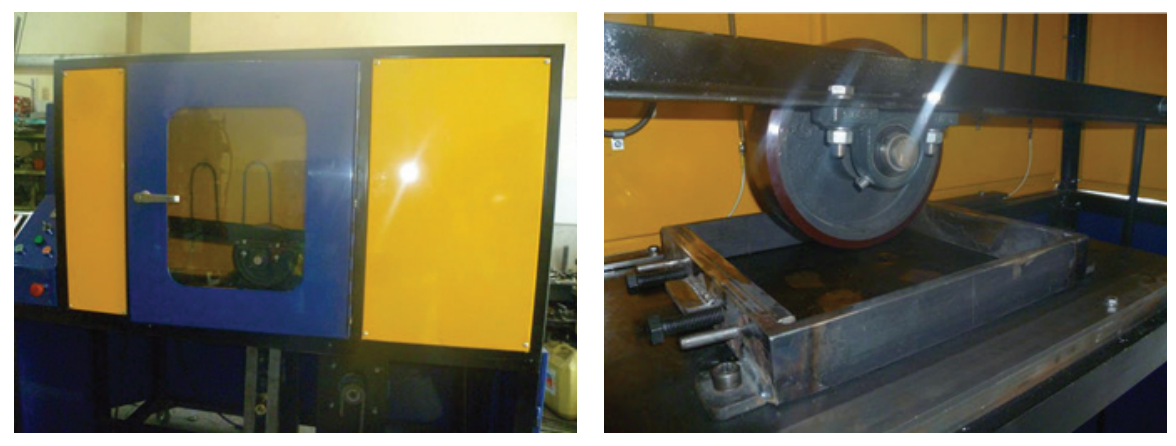

Figura 7. Equipo construido según norma INVIAS 756-13 para la determinación del ahuellamiento de mezclas asfálticas?

\section{$\mathrm{RAP}+\mathrm{GCR}+\mathrm{ECRL}-1$}

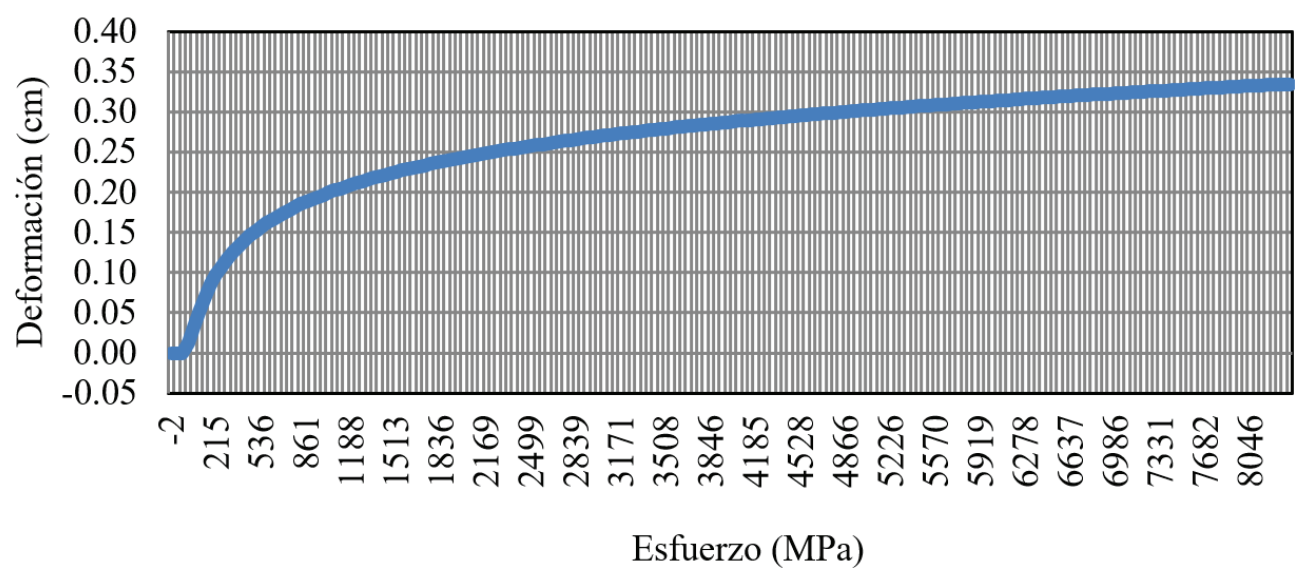

Figura 8. Deformación plástica de la mezcla con RAP modificado con GCR y ECRL-1

\footnotetext{
${ }^{1}$ Equipo financiado por la Vicerrectoría de Investigación, Universidad de la Salle,2015.
} 


\section{CONCLUSIONES}

Una de las variables que tuvo mayor influencia durante la consecución del RAP fue el contenido de ligante presente en la mezcla envejecida. Este parámetro es esencial para garantizar la homogeneidad del RAP seleccionado.

Debido a las diversas granulometrías que se consiguen durante los procesos de reciclaje in situ, la selección del RAP debe ajustarse a una granulometría para su reutilización, lo cual implica contar con la adición de material en los tamices en los cuales no se da cumplimiento a la granulometría. En este caso, la adición fue de arenas y material fino para quedar en la franja de la mezcla de diseño nueva tipo MDF-25.

El ensayo de fatiga para la mezcla de RAP completamente virgen y sin ligante no se consiguió, dado que por los procesos de envejecimiento la adhesión se pierde completamente. Debido a esto, se realizó un ajuste con emulsión CRL-1+GCR.
Comparando la ley de fatiga para la mezcla con 100\% RAP y la mezcla RAP+GCR, se evidencia que los resultados de la adición de emulsión CRL-1 y GCR, mejoran considerablemente los resultados de deformación de las mezclas con pavimento reciclado.

La deformación plástica para la mezcla RAP modificada con emulsión CRL-1 y GCR, da como resultado en esta primera etapa, 0,30 cm en promedio de las seis briquetas. Sin embargo, este valor se debe contrastar con un mayor número de muestras y diferentes dosificaciones de asfalto residual.

\section{AGRADECIMIENTOS}

A la Universidad de la Salle por el apoyo financiero para esta investigación, a la empresa Incoasfaltos y al Instituto de Desarrollo Urbano-IDU por su contribución con los materiales para esta investigación. Al ingeniero Fredy Reyes por su asesoría para la construcción del equipo según norma INVIAS 756-13.

\section{REFERENCIAS}

Espinosa Rodríguez, D. A. (2009). Guía de mantenimiento para pavimentos asfálticos de vías locales en Bogotá. (Tesis de grado). Universidad de la Salle, Bogotá.

Federal Highway Administration (2011). Reclaimed asphalt pavement in asphalt mixtures: State of the practice (No. FHWAHRT-11-021). Recuperado de: https://rosap.ntl.bts.gov/view/dot/40918

Federal Highway Administration (2019, Mayo 13). Asphalt Pavement Recycling with Reclaimed Asphalt Pavement (RAP). Recuperado de: https://www.fhwa.dot.gov/pavement/recycling/RAP/

Fernández del Campo, J. A. (2003) Recycling in Road Pavements, Third International Symposium on Maintenance and Rehabilitation of Pavements and Technological Control. University of Minho, Guimaraes.

Figueroa, A. S., Velásquez, R., Reyes, F. A., y Bahía, H. (2013). Effect of water conditioning for extended periods on the properties of asphalt binders. Transportation Research Record, 2372(1), 34-45. doi: 10.3141/2372-05

Herrera, O. A., Velásquez, C. E. D., Infante, A. S. F., Gómez, W. D. F., y Lizcano, F. A. R. (2013). Análisis de la alteración del asfalto colombiano en inmersión en agua. Ingeniería y universidad, 17(2), 427-442.

Hsu, T., y Tseng, K. (1996). Effect of rest periods on fatigue response of asphalt concrete mixtures. Journal of Transportation Engineering, 122 (4), 316-322. doi: 10.1061/(ASCE)0733-947X(1996)122:4(316)

Illinois Center for Transportation (2007). Reclaimed Asphalt Pavement - A Literature Review (FHWA-ICT-07-001). Recuperado de: http://hdl.handle.net/2142/46007

Lundstrom, R., Di Benedetto, H., y Isacsson, U. (2004). Influence of asphalt mixture stiffness on fatigue failure. Journal of Materials in Civil Engineering, 16(6), 516-525. doi: 10.1061/(ASCE)0899-1561(2004)16:6(516) 
Masad, E., Muhunthan, B., Shashidhar, N., y Harman, T. (1998). Aggregate Orientation and Segregation in Asphalt Concrete. Application of Geotechnical Principles in Pavement Engineering, Proceedings of Sessions of Geo-Congress 98. American Society of Civil Engineers, Boston.

Minnesota Department of Transportation (2002). Investigation of recycled asphalt pavement (RAP) mixtures (MN/RC - 2002-15). Recuperado de: http://hdl.handle.net/11299/758

Norma NFP 98 261-1. Test relating to pavements - determination of the fatigue resistance of bituminous mixtures - Part 1: Two points flexural fatigue test with constant displacement on trapezoidal isocel specimens. Association Francaise de Normalisation, 2001.

Norma I.N.V. E-201-07. Muestreo de materiales para la construcción de carreteras. INVIAS, 2007.

Norma I.N.V. E-440-13. Mezcla densa en frío. INVIAS, 2013.

Norma I.N.V. E.-756-13. Resistencia a la deformación plástica de mezclas asfálticas mediante la pista de ensayo de laboratorio. INVIAS, 2013.

Ostos, L., Duarte, J. M., Vargas, G. L, Camacho, J., Reyes-Ortiz, O. J. (2012). Comportamiento de mezclas asfálticas del Instituto de Desarrollo Urbano con adición de pavimento reciclado. VI Jornadas de Pavimentos y Mantenimiento Vial. Bogotá.

Qin, Q., Schabron, J. F., Boysen, R. B., y Farrar, M. J. (2014). Field Aging Effect on Chemistry and Rheology of Asphalt Binders and Rheological Predictions for Field Aging. Fuel, 121, 86-94. doi: 10.1016/j.fuel.2013.12.040

Rondón, H., Reyes, F., Gónzalez, L., y Vásquez, S. (2012). Ahuellamiento y fatiga en mezclas asfálticas. Bogotá, Colombia: Ecoe ediciones Ltda.

Strategic Highway Research Program (1990). Summary report on fatigue response of asphalt mixtures (No. SHRP-A-312). Recuperado de: http://onlinepubs.trb.org/onlinepubs/shrp/SHRP-A-312.pdf 